NISTIR 8095

\title{
Electronic Health Record Design Considerations in Responding to Incidences of Highly Infectious Diseases: Clinical Workflows and Exception Handling
}

Svetlana Z. Lowry

Mala Ramaiah

Emily S. Patterson

Paul Latkany

Debora Simmons

David Brick

Michael C. Gibbons

This publication is available free of charge from: http://dx.doi.org/10.6028/NIST.IR.8095

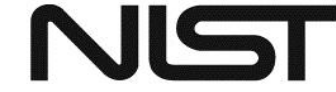

National Institute of Standards and Technology

U.S. Department of Commerce 


\section{Electronic Health Record Design Considerations in Responding to Incidences of Highly Infectious Diseases: Clinical Workflows and Exception Handling}

\author{
Svetlana Z. Lowry \\ Mala Ramaiah \\ Information Access Division \\ Information Technology Laboratory \\ Emily S. Patterson \\ The Ohio State University \\ Columbus, $\mathrm{OH}$ \\ Paul Latkany \\ New York Eye and Ear Infirmary \\ New York, NY
}

Debora Simmons

St. Luke's Health System

Houston, TX

David Brick

NYU Langone Medical Center

New York, NY

Michael C. Gibbons

The Johns Hopkins University

Baltimore, MD

This publication is available free of charge from:

http://dx.doi.org/10.6028/NIST.IR.8095

December 2015

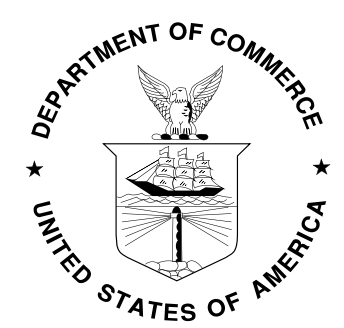

U.S. Department of Commerce Penny Pritzker, Secretary

National Institute of Standards and Technology Willie May, Under Secretary of Commerce for Standards and Technology and Director 


\section{Acknowledgments}

The authors gratefully acknowledge the intellectual contributions from the following reviewers:

- Christina Liscynesky, MD, Infectious Diseases, Ohio State University

- Courtney Hebert, MD, SBS-Biomedical Informatics, Ohio State University

- Harold Horowitz, M.D., Bellevue, NYU

- Margaret Price, MD, Infection Control at CHI Baylor St. Luke's Medical Center \& CHI St. Luke's Health System

- Dr. Layne Gentry, MD, Infection Control at CHI Baylor St. Luke's Medical Center \& CHI St. Luke's Health System

- Firas Zabaneh, MD, Infection Control at CHI Baylor St. Luke's Medical Center \& CHI St. Luke's Health System

- Ramani B. Reddy, MD, Infectious Disease Specialist, Holy Cross Hospital and MedStar Montgomery Medical Center 


\section{DISCLAIMER}

Certain commercial entities, equipment, or material may be identified in this document in order to describe a concept adequately. Such identification is not intended to imply recommendation or endorsement by the National Institute of Standards and Technology, nor is it intended to imply that these entities, materials, or equipment are necessarily the best available for the purpose. 


\section{Table of Contents}

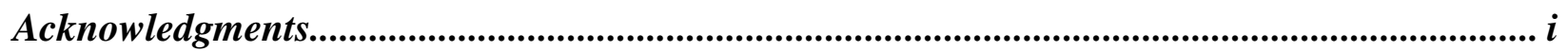

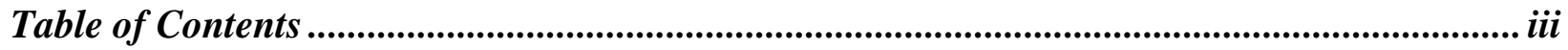

Executive Summary ........................................................................................................................ iv

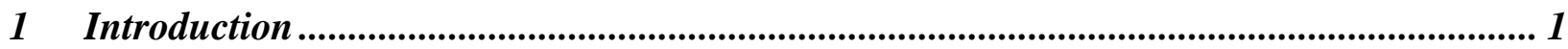

2 Methodology for Discussions with Subject Matter Experts ........................................... 3

3 Guidance for Improving EHR Support for Highly Infectious Diseases ........................... 4

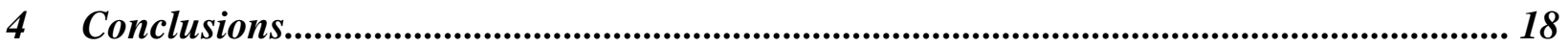

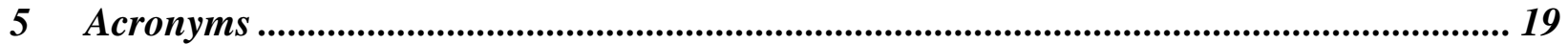

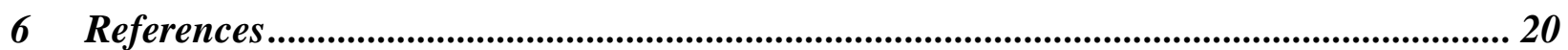




\section{Executive Summary}

Adoption of Electronic Health Record (EHR) systems in all care settings has become widespread. EHRs can support and revolutionize the way that public health is protected in the event of an outbreak of a highly infectious disease. In particular, EHRs can support continuity of care across transitions to specialized care settings, such as treatment for Ebola, by providing access to prior medical history, treatments, lab results, and other critical information to confirm or rule out an infectious disease and safely treat patients without exposing clinical staff and the public unnecessarily to infection.

In this report, infectious disease experts provide insights about patient safety and workflow considerations that arose during their experiences treating patients suspected of having Ebola. These considerations pertain to poorly supported tasks when treating any highly infectious patient. Highly infectious diseases include, but are not limited to, Ebola, measles, antibiotic-resistant infections (e.g., Methicillin-resistant Staphylococcus aureus (MRSA)), and mutations of influenza that are anticipated to occur in the future (e.g., H1N1 mutations).

The insights of the subject matter experts identified a wide range of opportunities to increase public safety by augmenting existing EHR functionality. These opportunities include:

- Identifier that says that patient might have a highly infectious disease;

- Alerting staff who are not providing clinical care to not access charts;

- Directly accessing 'read only' information from a prior organization about past medical history, labs, treatments;

- Obtaining an accurate immunization history without relying on patients to provide information;

- Having an "at a glance" summary of the status for an infectious patient;

- Correctly placing transferred patients across organizations;

- Tracking stages of progress in evaluating whether an infectious disease is confirmed for an individual patient and/groups of patients;

- Early mass notification of laboratory results for health department officials;

- Reducing uncertainty about whether laboratory tests are completed or expected and results shall be shared immediately following a transition of care;

- Identifying new areas of concern/outbreaks/diseases;

- Learning about common and variable presentation of previously infrequent diseases during outbreaks;

- Handling of calls, requests, screening and monitoring updates, etc., during outbreaks;

- Avoiding "labeling" patients by getting them ruled out;

- Efficient public safety data entry when treating an individual patient;

- Meeting U.S. Food and Drug Administration (FDA) paperwork requirements for investigational drugs;

- Decision support for clinical workers regarding when to consult specialists;

- Tracking locations of hot spots for outbreaks; and

- Communicating to the public about seriousness of preventive measures during outbreaks.

These insights are a first step to create an effective infrastructure to better identify, diagnose, treat, and report infectious diseases in the United States. The lessons from this effort might also have implications beyond the treatment of highly infectious diseases. A primary insight is that users need reliable, relevant EHR information, including a summary of relevant history and an easily interpretable view of when vaccinations were provided against the patient's expected immunization schedule. In order to achieve this goal, there needs to be confidence that the correct patient's record is open, that the information in the 
record is accurate, and that information that is used in graphical representations in structured fields is complete and integrates with information from multiple sources, including data from other EHR systems. The EHR needs to be a reliable tool verified through standardized testing that optimizes clinical outcomes and public health awareness by giving the healthcare team comprehensive and trusted information that can be efficiently interpreted across role-based physical and cognitive workflow. 


\section{Introduction}

Adoption of Electronic Health Record (EHR) systems in all care settings has increased. EHRs can support and revolutionize the way that public health is protected in the event of an outbreak of a highly infectious disease. In particular, EHRs can support continuity of care across transitions to specialized care settings, such as Ebola treatment, by providing comprehensive, reliable, and efficient access to prior medical history, treatments, lab results, and other critical information to confirm or rule out an infectious disease and safely treat patients without exposing clinical staff and the public unnecessarily to infection.

In this report, several infectious disease experts provide insights about unique patient safety and workflow considerations that arose during their experiences treating patients with or suspected of having Ebola. These considerations offer opportunities to provide better EHR-based support when treating a highly infectious patient.

The clinical experience with treating patients who have contracted Ebola in the United States fortunately remains limited at this time. Nevertheless, it is prudent to increase the national preparedness for the next wave of highly infectious diseases in that learning in real time during an actual outbreak predictably would have negative consequences for public safety. As one clinician wrote who contracted Ebola while caring for others, "Ebola is frightening, not just because of its high fatality rate, but also because of how little we know about it. We cannot explain exactly what it does to our bodies, nor tell patients who survive it how it may affect them in the future... I understand the fear that gripped the country after I fell ill, because I felt it on a personal level. People fear the unknown, and fear in measured doses can be therapeutic and inform rational responses, but in excess, it fosters poor decision making that can be harmful...We all lose when we allow irrational fear, fueled in part by prime-time ratings and political expediency, to supersede pragmatic public health preparedness."

One identified area of opportunity for enhanced preparedness is to strategically add capabilities in the EHR that can more quickly identify and quarantine patients suspected of having infectious disease/s. Specifically, based on what has already been used in a hospital, ${ }^{2} 1$ ) the clinical workflow was redesigned with a supporting EHR-based screening tool to capture travel history and other screening questions for emerging infectious diseases such as Ebola at the point of entry; and 2) revised policies to establish a more rapid identification and isolation of at-risk patients based on the patient's chart in the EHR.

Worldwide, emerging and reemerging infections continue to challenge prevention and control strategies. The impact of both new and reemerging infectious diseases on human populations is affected by the rate and degree of spread across geographical areas, depending on the movement of human hosts or of the vectors or reservoirs of infections, predominantly by travel. ${ }^{3}$ For Ebola, as well as a number of other highly infectious diseases that are endemic to specific regions, a critical component of early identification is recent travel history by a patient in geographic areas where there is a likelihood of exposure. Recently, for Ebola, this was Guinea, Liberia, and Sierra Leone, but it is expected that this list would change fairly rapidly during an epidemic. Therefore, it would be prudent to take advantage of resources which maintain updated information such as the Public Health Information Network (PHIN) Vocabulary Access Distribution System (VADS), which includes a list of countries in the value set, "Countries Widespread Transmission Ebola Virus Disease (EVD)" (OID: 2.16.840.1.114222.4.11.7248). ${ }^{4}$,

Designing preparedness solely for Ebola would likely be a shortsighted strategy. Many highly infectious diseases share similarities in the potential for causing epidemics which threaten the health of the public, as well as clinical providers, particularly in hospital settings. For the purposes of this effort, the focus was 
generating insights for protecting against these highly infectious diseases. These diseases include, but are not limited to, Ebola, measles, antibiotic-resistant infections (e.g., MRSA), and mutations of influenza that are anticipated to occur in the future as zoonosis (e.g., H1N1 mutations). The treatment of infectious diseases is in itself a prevention measure, limiting or preventing transmission to others with personal protection, general public health measures, or immunologic approaches such as vaccination. ${ }^{5}$

When considering how to better prepare for public health risks, there might also be lessons that can be learned from data registries. Data from registries help inform how to support public citizens exposed to highly infectious diseases. Patient cohorts can be identified for investigation and intervention. With data registries, it has been recommended that appropriate data elements should be designed for access and entry of information from immunization registries with consideration for established data standards, common data definitions, and how patient identifiers will be used. 


\section{Methodology for Discussions with Subject Matter Experts}

The methods that were used for generating insights were one-hour telephone-based discussions with Subject Matter Experts (SMEs) from four hospitals who either cared for patients with Ebola or cared for patients suspected of having Ebola and other highly infectious diseases. Examples of questions were generated a priori and vetted with the entire research team in advance. These were used during natural flow points during the conversations and included:

1. Please tell us about your experience using EHRs with patients suspected or confirmed to be infected with Ebola.

2. Did you experience any problems, and how could the support be improved?

3. What worked well with the support provided by the EHR during that experience?

4. If you could customize this functionality based on your needs, how would you do that?

5. What would be helpful for a physician to know with a summary overview about a patient who is under their care for the first time after a transfer?

6. Would it be helpful to have an alert which indicates that people should not access this chart because they are at higher risk for not following Health Insurance Portability and Accountability Act (HIPAA) regulations?

7. Would it be helpful to have a remote log-in 'view only' option to access information in the original treating hospital?

8. What infectious diseases would you put into "high risk" similar to Ebola?

During the interviews, notes were taken by the interviewer, de-identified, and reviewed by the subject matter expert for verification and to encourage additional insights. Responses to the expected and emergent questions were grouped to identify emerging themes regarding poorly supported work processes with the EHR. Recommendations to address the emerging issues were generated by human factors experts and reviewed by the interdisciplinary team, including physician and nurse clinical experts. The recommendations for improving existing EHR functionality are provided in the next section along with the supporting excerpted data from the de-identified notes from the discussions. The order of the recommendations is based upon the agreed priority of the issue to be incorporated into EHRs and/or availability of specialized applications that integrate with EHRs. Peer-review experts provided additional insights on framing recommendations and prioritizing suggestions based on reviewing a prior draft of this report. 


\section{Guidance for Improving EHR Support for Highly Infectious Diseases}

\section{Identifier that says that patient might have highly infectious disease}

It would be useful to have an additional category of identifier displayed near other patient identifiers such as date of birth. This particular category of identifier would help in identifying a patient when there is the possibility that a patient has a highly infectious disease. The three categories to display are: suspected, confirmed, or ruled out (with nothing displayed in the typical situation). This user interface location is reserved for infectious disease, and this location would not include any noninfectious distinct disease identifiers.

Related statements based on current concerns from SMEs are:

- One expert from Hospital 1 stated: "Pandemic mutated flu as the top one (it doesn't really matter what mutation). Ebola, Measles, potentially bioterrorism like anthrax. MRSA and botulism would be lower priority. Flu does kill people even now, but it's not at the same level."

- Second expert from Hospital 1 stated: "Infectious diseases to consider are measles, chicken pox and pertussis. With some of these, vacation history might be important, travel history might be important. Perhaps have a field like: Have you been out of the country recently? Yes/No."

- Expert from Hospital 3 stated: "You always want to know as soon as possible whether a suspected patient has Ebola. You need to know whether you need to track all of the people who were in contact with the patient, and then who they were in contact with. But you don't want to get everyone alarmed if it's not Ebola."

- Expert from Hospital 3 stated: "There are really two categories for suspected patients. Patients who have malaria and have a travel history of the known areas are a higher risk. There are "marginal suspected" patients like someone who can't remember exactly where they were in Africa, or someone who throws up in the Emergency Department (ED) who went to somewhere else in Africa but not to the known risk countries. Patients who were on the same plane as an Ebola patient. If there is even a high suspicion, there is a process that begins as soon as you hit the door, you ask the travel history and get the patient into isolation. If someone is walking into the ED, the chances are high that the patient is not highly infectious."

- Expert from Hospital 3 stated: "The ideal would be to utilize the problem list. In theory, highly infectious diseases would be entered into the problem list as soon as they were suspected and labeled as SUSPECTED highly infectious disease. Then tags would be associated with the patient to alert to the possibility of having it. But some patients have 20 problems, and it could get buried in there. And often problem lists only contain confirmed diseases, not suspected diseases. We definitely only want three categories for a disease: 1) confirmed, 2) suspected, 3) not. Any more categories than that and we will lose the attention of physicians who may have only a few seconds to think about it. The important diseases where this would matter are measles, flu, MRSA, and SARS. And any diseases that we have not identified yet. Across the medical system, we have different patient populations. In the outpatient setting, we are less likely to encounter highly infectious populations, even though we have personal protective equipment (PPE) there in case. The walk-in emergency is the likely scenario."

- Investigator: "Would it be useful to have an identifier at the level of the Medical Record Number (MRN) that identifies that a patient is suspected or confirmed to have an infectious disease?" Expert from Hospital 3 stated: "I don't know. You would expect that information to be in the problem list. So you see it there, but sometimes it's not at the top or you might miss it. If a patient is confirmed to have the disease, everyone already knows it. If there's a high chance that they have it, same thing. With Ebola, they are physically separated, and it is nearly impossible to accidentally walk into the area where the patient is kept." 
- Expert from Hospital 4 stated: "If someone was treated for Ebola, it is not a public health concern once treated. Once past the transition period, not going to be a risk. Track the patient not infectious anymore, so I don't know how the EHR could support that. If want to flag used to have Ebola, more helpful to know had the infection so has to be careful but not worrying about spreading anymore."

- Expert from Hospital 4 stated: "Clostridium difficile (C.diff) outbreak in nursing home, need to know patients have C.diff. More helpful for these things than Ebola where there is only one or two patients to worry about."

- Expert from Hospital 4 stated: "Some patients with sexually transmitted diseases (STDs) and have internal flags who keep going from physician to physician and spread it and don't get treated. Can't flag the patient in the EHR to warn patient is a public health risk."

\section{Support for alerting staff who are not providing clinical care to not access charts.}

One expert, when directly asked, stated an interest to have a tailored alert warning clinical providers that there is a higher risk of termination of employment or other disciplinary measures when accessing information that is not required to perform their assigned care activities due to violation of patient privacy.

Related statements from SMEs are:

- Investigator: "Would it be helpful to have an alert which indicates that people should not access this chart because they are at higher risk of losing their job?" One expert from Hospital 1 stated: "It would be fine to have this. Healthcare employees should know not to access charts which they don't need to do their job... This should not happen, and there should be consequences if it does. It would be appropriate to have more tempting charts that have higher levels of warnings, but this would be a decision that the medical center would make, not the EHR companies."

\section{Support for directly accessing 'read only' information from a prior organization about past medical history, labs, treatments.}

Determining whether a patient has a highly infectious disease and generating an appropriate and safe treatment plan cannot be done in a vacuum. It is of prime importance that clinicians should be able to directly access all available information about the past medical history, including lab work that has been ordered and the results and treatments that have already been initiated or were planned. When asked to prioritize a subset of information, providers were resistant to the notion that a limited set of information could be identified a priori across all of the potential types of highly infectious diseases, and felt strongly that they needed all information that was potentially available. On the other hand, none of the SMEs felt that they needed to have "write" access to any of the information, but only "read only" access.

\section{Related statements from SMEs are:}

- One expert from Hospital 1 stated: "When I have taken over the care of a patient treated elsewhere, I will call the microbiology lab and get a report verbally on patients. Nearby hospital will ask me to sign a form. If anyone tells me that they can't give me information, then I talk with their supervisors and it gets worked out since I have assumed that patients' care and need access to it. When other hospitals have an EHR and use a software module to accomplish the same task, that works well too. For example, I can access the information at a hospital two hours away. I might not have access to the daily vitals, but I have pretty much everything that I need with the culture for infectious diseases (IDs), progress notes, and imaging reports. I mostly need lab work, the vaccination record (having 2 doses of measles, mumps, and rubella (MMR) means that the severity of illness with measles will be much less), whether or not the patient is pregnant or has human immunodeficiency virus (HIV), and their medications given at the prior hospital and what medications the patient took at home. If a patient is pregnant, then we need to know that. The 
emergency room (ER) screens all patients with a urine dip for pregnancy, but it does not reliably get into the EHR or is not easy to always see at a glance when a patient is pregnant. This affects everything because the patient could become more ill, some antibiotics we can't use, we probably can use some Tylenol to bring down the fever so that's less of an issue, but whether or not the patient can be intubated is also important to know. We need to know if a patient has been on chemo, and if so, the specific medications and dosages, what kind of cancer they have, and where it is. Not enough to know lung cancer - we need where exactly and what medications/approaches have been used to treat it. The medication list is often not as up to date as we need, particularly with respect to medications taken at home."

- One expert from Hospital 2 stated: "We receive patients, have a large outpatient clinic. As a receiving hospital, they come here first. What if a person got through screening with no fever (had ice in their mouth and lied) and then gets hit by a car and come to the ED room. Bleeding a lot and in an early stage with some Ebola in the blood. How do you - can't get a history? Treat them how? Guy in Africa had a heart attack, back within the prescribed period of time to develop Ebola, had a massive heart attack and died. Wouldn't have the history of exactly what happened. Travel is the key component to the most recent epidemic. If they are not having a fever or certain other symptoms, at that point, they're not highly infectious. If they fit in the window period (2-21 days) and they have traveled, and not having symptoms, comes into the ER, broke their arm, 7 days out from working in a country with people and some healthcare workers and somehow got through the system, that person is drawn up by the health department and has to call in twice a day with their temperatures, and go on a list, and if they didn't call, the health department calls them. Elaborate system, at least in our city. The thing that concerns me is that I'm more nervous as Ebola fades from our memory, if you don't tell the kid to brush their teeth, they stop. All this stuff going on in the airports, money cut and move somewhere else and less rigorous on checking people. Maybe racial profiling - white rich guy didn't work with them and lets them through. So more worried that we're going to have an error."

- One expert from Hospital 3 stated: "You do the test to see if there is Ebola (blood test) and then send the patient to a receiving hospital. You don't want word to get out that there is an Ebola patient, because then all of the elective surgeries go to zero. Other hospitals experienced this, and some patients can be harmed by delaying necessary surgeries, plus the financial loss to the hospital."

- One expert from Hospital 3 stated: "The biggest remaining gap in EHR functionality is in hospital to hospital EHR information sharing, even when the hospitals are both using EHR. Also city to city sharing. Someone goes to another hospital's walk-in clinic and they call for us to come to take the patient to provide care. It slows everything down not to have the history. If you know a patient is highly infectious, then it's a phone call to the on-call infection control person but if you don't have access in the ED to the prior patient records, that's a problem. You want the patient data demographics in our system to treat the patient. Maybe this is too simple, but ideally you would have a flash drive taped to your forehead as a patient that you take with you with the key information. We need the history. If a patient goes unconscious, which is not at all unlikely, then you can't interrogate the patient. You need to know the travel history, the vaccination history, the history of symptoms in the recent past as well as whether they were experienced other times in the more distant past. The most critical piece of information is exactly what lab tests have been done and are pending and when they are anticipated to be done. With uncertainty, the receiving hospital is likely to just order them again to be sure, even when this creates additional risks for clinical personnel to draw blood, etc. But if they know where the blood has been sent and who to contact and what has been ordered, then they don't need to. Then we also need to know when exactly the isolation precautions began. In the ED? On admission to the intensive care unit (ICU)? Before the emergency medical services (EMS) arrived to take the patient to the hospital? How many hours was the patient in the ED before being seen? Or was the patient in the hospital for two weeks and then sent to us? Or in a hospital for two weeks, left, and then went to ED after 
that? If so, what status was the patient when they left the hospital at discharge? We need a medical summary of the ED visit. We need to know what lab work was done. We need the key elements. We had similar issues during Hurricane Katrina with patients sent here. Some needed dialysis and we didn't know their dialysis history. Some needed psych meds and we didn't know the history. Some we thought might have tuberculosis (TB) and wanted to know their TB history and vaccination history. We want a summary of key elements, if only as a printout."

- One expert from Hospital 3 stated: "Our branching is: Is the patient highly infectious (Yes/No). Then is isolation required (Yes/No). Then does travel and exposure history match with known risks (Yes/No)? Then physical findings: shock? diarrhea? lab, treatment (specifically need to know when fluids were started for Ebola which is supportive for shock), the nursing report blood pressure (BP) 160/90, pulse X, temperature X). What else is in the treated history for what people did and the thinking behind it."

- One expert from Hospital 3 stated: "The worst case is the patient who is unconscious in an ambulance with no family member or accompanying person. Or when there are language issues."

- One expert from Hospital 4 stated: "We do a lot of HIV. Prior history is very important, have no clue what they have been on before. It would be helpful to have vaccination history, prior medication history, and allergies to transfer from one physician to another. Some patients are knowledgeable, but some have no ideas what they have been. Don't think need to manipulate the information, don't want anyone to change my information from other places. It's something that someone else did, so I will create my own and need to get whatever I need. Read-only access is what is really needed. Confidentiality is always a priority and protect the information and some patients don't like that any physician can see they have HIV. Only want that physician to access the chart. Put a flag on the patient chart, have to type in a reason why you are opening it up. Patient care, quality improvement, whatever. Internal flag when you open a chart. Other physicians in the practice may not know that patient has something sensitive, so put in a small flag which can be accessed and removed at any time, and doesn't track that it used to be there. Pops up at the chart and can't get in there unless it types in your reason."

- One expert from Hospital 4 stated: "I have no way to search cultures even now. If it happens to be in the system or if it happens to be a system I have access to, all hospitals have thing where you can look at it. Some can get into places with basic information, it's a portal, with a list of patients, then can get their information. They do have discharge summaries; it might happen to be there. Depends on what the hospital includes in the summaries and depends on what is included in the portal."

- Investigator: "Would it be helpful to have a remote log-in 'view only' option to access information in the original treating hospital?" One expert from Hospital 1 stated: "Sure. Software module works well. I would like local competing hospital to sign an agreement to have software module integration since they are now on EHR. Our patients routinely go back and forth and we need that information. If the hospitals are not on EHR we use, we do not really have any way to share data other than verbally with phone calls or faxing information."

- Investigator: "Do you use any workarounds to highlight information that is potentially available in a patient's chart but might be missed without using them? For example, are additional communications used outside the EHR such as via email, phone, or pager systems?" One expert from Hospital 3 stated: "All hospital records have next of kin, and so if you have access to records, then you have access to that. In Texas, driver's licenses have some information for who to contact if someone didn't accompany the patient. Passport and phone numbers for travel if you know where they were flying from."

- One expert from Hospital 1 stated: "With psychiatric hospital patients, we have the ability to "break the glass" to see relevant information, and that is helpful and works fine." 


\section{Support for obtaining an accurate immunization history without relying on patients to provide information.}

Interoperability, when it exists across diverse data sources of immunization history, with the ability to merge information in a standardized, structured way, and make annotations on plotted values that are suspected to be inaccurate would be useful augmentations to EHR functionality. The immunization history tends to be limited solely to immunizations that were given within the same organization and only since the EHR was implemented. Many immunizations are given to patients before they are allowed to be cared for by hospitals that specialize in treating adult patients, since they are typically given in pediatric outpatient clinics. In addition, many patients live in more than one location, or receive immunizations at nontraditional care locations, such as urgent care clinics in the community. The importance of not having accurate immunization history has a higher patient safety impact because of highly infectious diseases such as measles.

Related statements from SMEs are:

- One expert from Hospital 1 stated: "Although this was not needed with those two cases, with other cases like with measles, the immunization history will be important. This is typically entered by the registered nurse (RN) based upon verbal report from patients. Generally, patients do not remember when they had them. For example, with pneumonia, there's an older vaccine and a newer one. If it was had more than 1.5 years ago, then we can guess that it's the old one. Patients will say "look at my record" but we only have something if it was given here. Some patients are savvy and bring a printout of everything that they have ever had with vaccination history with exact dates, but not everyone knows to do this. If you are an employee of the hospital, in the Employee Self-Service/Employee Health area, you can get a record of all of the vaccinations you have had while employed."

- Second expert from Hospital 1 stated: "Lack of information on vaccines in adults is a problem, especially if we are concerned about a vaccine-preventable disease like measles. If a vaccination was done at our hospital, it's in the chart. Otherwise it is just documented in free-text, if at all. We should have a more robust way to document and display the vaccines given within a health system. Ideally, we'd have a statewide vaccination database that we could access."

- One expert from Hospital 2 stated: "One of the things I found out from pediatricians involved in vaccination log at the health department by clinics that give vaccinations, found out all the people had been vaccinated at a city clinic because we're a city hospital. Private doctors don't enter their stuff into this. Who in our clinic had been appropriately vaccinated, so didn't have to deal with those people urgently. A better system of tracking immunizations applies to pertussis, measles, and rubella. Not as much from abroad. Probably aware that the influenza vaccine this season was less important since not matching the vaccine. Good to know if vaccinated for influenza but kind of ignored it. Pneumococcal vaccine. We need a database in the United States to track vaccinations. There was a study published many years ago where they ran a travel clinic and asked people about immunization status for various things applying to a travel, didn't know last tetanus shot, didn't know pertussis and didn't know most vaccination history. So person comes in with pneumonia, don't know history, could see if had pneumococcal vaccine if they had documented vaccination, it would be lower on the differential. The city health department for children has a childhood program where they have all that data but only for people who go through the program, their data goes there. This electronic medical vaccination program through the city is very helpful."

- One expert from Hospital 2 stated: "Vaccination history, need to have this if just out of pediatrics, two years later go to a different system, most of the time we end up titers to see if they have vaccinations or not. Pneumovax given multiple times in some cases since can't remember." 


\section{Support for having an "at a glance" summary of the status for an infectious patient.}

Having the ability to quickly gain "situation awareness" about the status of a patient suspected or known to be infectious would be useful in delivering effective care in an efficient manner. Although none of the SMEs suggested this functionality to be needed based upon their experiences to date, as the number of infected or potentially infected patients increases during a pandemic, this strategy is anticipated to be more important than what has been experienced to date. One SME defined what would be useful to contain in a summary overview when directly asked about this idea.

Related statements from SME:

- One expert from Hospital 1 stated: "A helpful summary would include the most recent lab values, what medications were taken at home, what medications were given in the transferring hospital, most recent vitals, whether on oxygen, whether on the ventilator, if pregnant, or HIV status."

\section{Support for correctly placing patients transferred from the outside in coordination with other organizations.}

Tailored EHR support for correctly placing patients transferred from external entities by a "bedmaster" or other providers who are authorized to make placement decisions for potentially infectious patients is recommended. Since it is unlikely that processes for placing all patients will accurately identify high-risk patients for spreading infections in all cases, it is suggested that this support be provided for all placement decisions. Correct disposition of patients to the justified location is important for all hospital patients. For highly infectious disease patients, there is unnecessary exposure of additional patients and clinical workers when this does not occur, and there needs to be an additional transfer internally following the intake.

Related statements from SMEs are:

- One expert from Hospital 1 stated: "Not with these patients, but with other ID patients, we have had problems where the outside call to transfer patients in gave information during a phone call for the transfer which did not indicate as high a level of care as they needed. For example, floor patients would really need to be in stepdown. Stepdown would really need to be in the medical intensive care unit (MICU). Because nurses can't rotate like physicians have, that necessitates an additional transfer for appropriate care. In particular, having the most recent vital signs would help with correct placement."

- One expert from Hospital 1 stated: "The Regional Trauma System helped with triage for what patients go where who have botulism. That worked pretty well."

- One expert from Hospital 1 stated: "For measles, you do not have a different unit like Ebola to care for patients, but you do have to have them in negative airflow rooms, which are special. If not vaccinated with measles, mumps, and rubella (MMR) vaccine, 9 out of 10 people will get measles if they are in the same room without protections, rarely, they can get encephalitis and die, so it's very important to protect people by using the negative airflow. Our rooms alarm if the pressure changes, and so the negative airflow is maintained well."

- Expert from Hospital 3 stated: "We want early detection for potentially highly infectious patients. Have them in a single room as the first line of defense, then cohort them when that is possible. In the ER, ICU, everywhere once a known respiratory issue, then put patient in negative pressure as quickly as possible. If you have the ability for syndromic surveillance, then fever and rash are the two biggies to track. Also respiratory issues. Then mental status since brain turns to mush with many highly infectious diseases. It's a double whammy because 1) you cannot get a history from the patient directly; and 2) any infection in the brain likely has gone throughout the body in all the important organs. You want to know if the patient has diarrhea or other symptoms." 


\section{Support for tracking stages of progress in evaluating whether an infectious disease is confirmed for an individual patient and/or groups of patients.}

EHR 'status boards' for tracking progress in treating multiple suspected or confirmed infectious disease patients is recommended as an opportunity. Similar functionality is employed in Emergency Operations Centers, such as for tracking wounded during a hurricane.

Related statements from SMEs are:

- One expert from Hospital 1 stated: "If we had a patient that was actually suspected of having Ebola, then we would keep them here for evaluation with polymerase chain reaction (PCR) testing. If PCR was positive, patient would be transferred to a hospital two hours away. If negative, we would admit the patient and repeat PCR in 72 hours. If second test is negative, we would release them from the Ebola unit."

- One expert from Hospital 2 stated: "Not even be tested for Ebola. Do all the precautions as if have Ebola and watch them for 24 hours or 48 hours with precautions and if don't act like Ebola, they go home. One person came in very low risk and didn't even take their blood. First person from the beginning with no blood."

\section{Support for early mass notification of laboratory results for health department officials.}

Although all of the SMEs emphasized the importance of directly communicating by phone with health department officials as opposed to electronically based communications, several of them identified areas where earlier notification of specific findings would be useful, such as lab results being shared with the ordering providers, infectious disease experts at the organization, as well as identified health department officials at the same time.

Related statements from SMEs are:

- One expert from Hospital 1 stated: “Typically during a situation where it's needed, the health department will answer questions about patients. When there was a mumps outbreak and a botulism situation recently, we had daily conference calls (verbal only) with them and that worked well."

- One expert from Hospital 2 stated: "People calling into the health department with temperatures first contact we had had is with the health department. They call us and say we have a case. Fire Department calls the health department, who calls them back. Or another hospital to get to us. Very specific system. To get to our hospital goes through the health department. If someone admitted to hospital, they fit the right characteristics. Stay in hospital, too low a risk. If strong enough risk, then we will get a call. A whole call tree. It is a very elaborate setup."

- One expert from Hospital 3 stated: "The first patient was at a different hospital. The ER physician recognized that the patient had recently traveled from a country. On Sunday night, the patient had flown into the city and then gone into the ED with the fever of 102. Infection control was called and we went from there. Well, actually a lesson learned was that he called everyone but us first the health department (couldn't remember if it was for the city, county, or state) and the Centers for Disease Control and Prevention (CDC). Now people know to call our area, Infection Control, first, and then we talk with epidemiology and how to report it. We are the ones who know who to communicate with and how and have developed relationships with them."

- Expert from Hospital 3 stated: "The city and state health departments want to know right away, also the commissioner at the health department."

- One expert from Hospital 4 stated: "Lab automatically sends results to health department. That is a nice feature. Has nothing to do with the EHR, it is the lab that has a list of reportable infections. Legionnaire, salmonella, these things, and then they call the patient and try to find out where it came from to see if there are more cases happening someplace. Recently had quite a few cases and pointed to some hotels in the area and things like that." 


\section{Support for reducing uncertainty about whether laboratory tests are completed or expected and for results to be shared immediately following a transition of care.}

Real-time display of received laboratory results in expected places within the user interface for clinicians even when tests are done by specialized laboratory facilities that handle highly infectious samples would be highly useful. Given the unique risks to clinical workers from obtaining blood samples for laboratory tests, avoiding redundant labs following a transition of care in the case of uncertainty about whether a lab test was ordered as well as whether results will be shared immediately upon receipt with the new organization now treating the patient is of central importance. In addition, the testing of the samples themselves can introduce risks to all clinical and laboratory workers handling or exposed to the specimen, and so only a small number of specialized facilities perform these. Therefore, having targeted support for receiving results quickly and reliably from external testing organizations is recommended. With some EHRs currently in use in hospitals, results from external testing organizations that are received by fax and then scanned as PDF formats are located in folders labeled with phrases such as "media" that are infrequently viewed by physicians. Having critical information stored in a rarely viewed folder rather than in an expected display of returned lab information on a dedicated tab would increase the risks of substantial delays or not realizing that the results have been received.

\section{Related statements from SMEs are:}

- One expert from Hospital 1 stated: "For Ebola, we would use a portable machine for labs. It prints paper and nurses can manually type in the information. We haven't had it in a real situation, but I think that will work fine."

- One expert from Hospital 2 stated: "We're not doing the Ebola test here because we are across the street from the city health department. We run it across. This is a many-step process---to get blood from patient on the unit across the street to have it run. Elaborate system. Results don't come back through the EHR. The results come back through the doctor's notes. Just call the lab. Most of the centers doing this now have a lab on the unit itself. We try as much as we can to screen people out very quickly and get up to the unit where the lab is. Results do get into the EHR. If we get a history in the ED or in a clinic that a person has traveled and has fever. If no fever, call the health department and ask if want to watch from home and bring in if have fever or admit to the unit. If person is screened by someone (greeter if not a nurse), fever or bad diarrhea and they have traveled to one of the Ebola countries, they will be put in an isolation room where no one goes in (in the ER). Interview to ascertain the history is correct through a cellphone or phone in the room. Gets a little tricky if an interpreter is required. No blood taken in the ED. No one gets donned and doffed unless has to go in the room. People come down once it's confirmed that it's a high-risk patient. That whole series of events, people who come in are already gowned and ready to take to the unit, which has been activated. Nothing even in the EHR."

- One expert from Hospital 3 stated: "The patient was here for a week but on day 4 they finally did a malaria smear, which came back positive. They had one ordered earlier, but they were trying to avoid doing unnecessary tests based upon the 2002 CDC guidelines, which have since been updated. With a blood test, the worry is exposing the clinical workers. Not just the nurses taking the blood sample, also what lab equipment to use was a question. State health department wanted to send the blood to a special place and the CDC, but the lab was closed so it went somewhere, I can't remember where. We don't deal with blood with possible Ebola here, so the question was where to send it at the time? Now we know to send to a specific place in another city."

- One expert from Hospital 3 stated: "In some cases, you likely want to do a screening lab. Might have to do a lumbar puncture."

Support for identifying new areas of concern/outbreaks/diseases. 
EHR surveillance and associated alerting support for identifying emerging areas of outbreaks for infectious disease specialists at hospitals would be useful.

Related statements from SMEs are:

- One expert from Hospital 1 stated: "For the infectious disease department, it would be nice to have some way to be alerted when there are identified areas of new concern. For example, 3 weeks ago there was an outbreak of botulism in a nearby hospital. The neurologist had 3 patients and identified it pretty quickly, so only one died. There is a risk that we would not know about these kinds of things for some hospitals that are nearby. It would also be helpful to receive alerts from the CDC if there is information about a pandemic flu, which is probably our biggest worry that would pop up information updates when you log in to the EHR. In theory, Homeland Security or other agencies could similarly inform you about bioterrorism issues such as anthrax, although that seems less likely to happen."

- One expert from Hospital 3 stated: "Now we have a hard stop so no one can skip a question, but as the risk goes down, we'll be removing the hard stop, since then it will be viewed as an extra step that is not necessary. Our rule is that as long as there are cases in the area, we'll keep it, and then we'll dump the screening tool when the risk lowers. It's a simple change that our local IT people can do, it doesn't require an EHR build. We can use this approach for all highly infectious diseases."

- Investigator: "If you had to guess, which infectious disease do you think has the potential to wipe out a substantial portion of the human race in the future?" One expert from Hospital 3 stated: "Well, a lot of folks talk about multidrug resistance (MDR). I could see it, but it's not at the top of my list. Mostly it shows up in patients who have been on antibiotics for years. It tends to be where patients have flora in their gastrointestinal (GI) tracts and have had it already before they come into the hospital. For me, my money is on the flu as a mutation, either H7 or H9. It's deep in the lungs. It's highly infectious before you have symptoms. It's always mutating. It is likely to become resistant to the antivirals that we have now. We're likely to have shortages of medications, even if they are effective."

\section{Support for learning about common and variable presentation of previously infrequent diseases during outbreaks.}

Emergent outbreaks for infrequent infectious diseases where there is not recent hands-on experience could be supported by images and video that represent the range of how the diseases present for both infectious disease specialists and frontline providers in order to support timely diagnosis and treatment.

Related statements from SMEs are:

- One expert from Hospital 1 stated: "With measles, many of us are too young to have personal experience with a case. If there is an outbreak, perhaps there could be a way to support learning about how to better recognize patients as having measles via the EHR."

- One expert from Hospital 1 stated: "With Middle East Respiratory Syndrome (MERS), it's a new virus and we really don't know that much about it. From Saudi Arabia, it was mostly men on dialysis, but it's unclear how cultural factors played into that."

Handling of calls, requests, screening and monitoring updates, etc., during outbreaks.

It is important to reduce unnecessary calls and requests that are unlikely to actually be cases of infectious disease. With Ebola, this primarily relates to patients that have a travel history in Africa but not the specific geographical areas of concern. With Ebola as well as with other infectious diseases, patients lacking troubling symptoms also are unlikely to be infected and highly infectious, such as when there is no fever in a patient suspected of being infected with Ebola. 
Related statements from SMEs are:

- One expert from Hospital 1 stated: "We have not had any confirmed Ebola patients treated here, although we are the receiving hospital in the area if there are any. We had two cases where the nurses called me (infectious diseases) because they were concerned, but they weren't really candidates for ruling out Ebola with a blood test as it was so unlikely. One patient was a lady from a country with no Ebola who was treated in an outside hospital in the surrounding rural area. She was elderly and had high fevers and returned very sick from acute HIV. She was transferred to our hospital on Sunday, and the nurses were concerned that she was being treated on the regular floors with other patients. But countries like country are not really an area of concern for Ebola. Another patient had been in a country with no Ebola. The patient tried to commit suicide and had belly pain, but did not even have a fever. The patient ended up in the psychiatric ward, and the nurses were very concerned over the weekend. They called us multiple times (infectious diseases) and then they called the medical director, who called us, and we explained that there was no concern."

- One expert from Hospital 1 stated: "If a patient is from Africa, such as from a country, we can maybe reduce ER calls to ID by letting ER physicians know that the patients from a country are not from Guinea, which is the area of concern. Perhaps this could be educational information about Ebola that is accessible via the EHR or answers to FAQs or something like that. Right now, we are using whether or not someone went to Africa to know if they are likely to have gotten Ebola, but eventually the disease may spread, probably to places like New York, D.C., Texas, California first before the Midwest. Then we will want to track where patients caught it differently, and also change the screening alerts to update that new location information."

- One expert from Hospital 2 stated: "How much are we as a society trying to use technology to prevent errors and to help physicians in the medical system improve care and reduce risk? With something like infectious diseases, move off of Ebola, one of the questions becomes where do you enter that? Person has a fever. That flags a series of questions or issues. With travel history? One of the big issues that I find and talk about this with people I work with, is the issue of the EHR has become some kind of a quagmire since so many lobbyists. Smoking people, and can't have smoker to have 15 questions. To get through the EHR can be hazardous if a person has pain. All done by forced fields so far the solution. Forced field could be travel history because people don't ask it routinely. With advent of Ebola recently and with SARS and MERS (Middle East Respiratory Syndrome), with the advent of these, the notion of taking a travel history has really gotten prominence. In our hospital, not sure if done in the city. One question would be: is there a place in the EHR for a travel history to actually have a separate one distinct location within the user interface - it's frequently included under the social history and not separate. If a travel history, then a series of questions. Again, if it's a forced field, then another field to go through, but could serve as a reminder to everybody. Whether it's put in the nursing field or doctor field, certainly in the ED could have a field like that. One could approach it from fever. In terms of the notion of infectious diseases and transmissibility with measles to stop an outbreak, no one is screening for rash and fever. If fever comes up, a series of questions in the right moment could indicate that a person has measles. Same for pertussis. Diseases require isolation and in the ED, or a doctor's private or hospital clinic, could reach havoc. Several centers in the United States supposed to deal with Ebola. Some like the StateX unit, it's really unlikely that someone will find out they have Ebola. Most likely picked up at the airports now. A major screening process at the major airports, Chicago, NYC, and if anything, if you want to pick up a lot of people, make the test very sensitive but not specific. People coming into city airports with a fever and very low risk for Ebola, they are being sent to our hospital. From the minute they're identified, it's craziness. The fire department gets involved transporting patient with equipment. Once at hospital, there's an incident command alert and people rush in. Not unique. We get people from a company hired to take care of the trash, from Boston last time, emergently. Costs an enormous amount of money. Now the health department, says every time this happens, can't make it quite so sensitive. That's 
a long-winded answer, and that is most people really picked up in the airport and at least in city. Everyone asked. People greeting patients to ask about a travel history and a fever. If a fever and every travel history. Asked about Ebola region and the Middle East for MERS. Measles. Last year woman came in pregnant with measles and no fever and no rash. A day later developed a rash. No way picked up in the obstetrics (OB) clinic. Hours each day to deal with the health department and people called back in to see if they have titers. I had to manually go through the scheduled list of patients who actually visited the clinic at the same time. It was very laborious. Pregnant women. Active screening process. Health and hospitals. Major cities have signs in multiple languages asking if you had a travel history."

- One expert from Hospital 2 stated: "Honestly don't think a lot of people need to know real-time the answers. Screen is very sensitive but not particularly specific. So we have people come in with a psychiatric illness who say I touched a person who was living with a person who was in Africa and that person with the psych illness got put in isolation until we all agreed that he had no risk, but psychiatric problems."

- One expert from Hospital 2 stated: "Where the EHR can be helpful - screen savers to remind people. Do people continue to look at the screen savers? Could be designed a lot of ways - has your patient been to Africa? Don't forget to ask travel history."

\section{Avoiding "labeling" patients, as in Ebola, by getting them ruled out.}

Three SMEs highlighted a trade-off between protecting public health by ruling out a highly infectious disease for a patient and unnecessarily escalating public dread and negative impacts to patients from being labeled as suspected to have the highly infectious disease.

Related statements from SMEs are:

- One expert from Hospital 1 stated: "For the second patient, I waited for four hours before I decided that I needed to write a progress note making it clear that there was no Ebola because I didn't want the patient labeled as an Ebola patient unnecessarily, but after the call from the medical director, I felt that it was necessary. It would be nice to explicitly have a way to indicate that a patient has been evaluated for whether they have Ebola and that we are not concerned to not only people involved in the care, but also people peripheral to the care who should not be looking at the progress notes in the chart. For example, we could have a field for high-risk infectious diseases that distinguishes suspected, confirmed, and ruled out. Ideally "ruled out" would be something more like "evaluated and we are not concerned," but we have been using the phrase ruled out, so if you need something shorter, that would work."

- Investigator: What do you think about having the ability to have a different name displayed than the patient's name to make it harder for the "wrong" people to access the chart for someone who has been reported in the media to possibly have Ebola? One expert from Hospital 2 stated: "I don't know that that is the EHR's job, but there are all these Health Insurance Portability and Accountability Act (HIPAA) violation laws. If you go into a chart that you're not caring for, it's a HIPAA violation. With an Ebola case or a famous person, everyone wants to look at the chart so they might change the name. Certain criminals, institution changed the name so that they couldn't go on. Institutional thing. Don't know how the computer would recognize unless there was a certain disease group with pop-up to consider changing a name because of HIPAA."

- One expert from Hospital 2 stated: "Clinical staff would be fired if anyone put out YouTube videos of patients. One weekend I couldn't come in one day and someone covered for me and left a note in the chart. Another ID doc works in the group got a call why did my covering person have a note in the chart? Were they involved with the care? Who is this person? Really went through all the people who clicked on that person's chart and people got warnings. Some people found out what the name was and wanted to look and see what was going on. People will be dismissed if there is inappropriate chart access by an individual." 
- One expert from Hospital 3 stated: "We had 5-6 patients who we suspected had Ebola. None of them actually had Ebola, we had no confirmed Ebola patients. Three of them were much more possible than others, including one who had malaria. Some were pretty clearly unlikely to have it, like they had kissed someone who had recently been "somewhere in Africa" and had a fever. One was an airport employee who clearly didn't have it, but was worried about it. There the issue was to avoid public panic that was unnecessary. One was a challenge because it was a lady who was pregnant who was suspected to have malaria. There was a family member who took a picture with a cellphone of the folks with protective gear on who came to get a patient, and we got a call from the press asking about the patient. Our goal there was to keep off the media. We have corporate communication people. We need to keep information confidential in order to meet HIPAA requirements."

- One expert from Hospital 3 stated: "All patients in the ED are screened for Ebola. If blood tests are required, take them quickly and then get the patient to a small hospital. If folks know that there is a possible Ebola at our hospital, nearly all elective surgeries will be cancelled. The perceived risk is typically higher than the actual risk in that situation."

\section{Support for efficient public safety data entry when treating an individual patient.}

One SME suggested optimizing the efficiency of data entry for data relevant to an epidemic. The suggestion was that missing data would be reduced if entry could be made more efficient for frontline providers who do not directly benefit from entering the data.

Related statements from SMEs are:

- One expert from Hospital 2 stated: "In many ways, the EHR makes things a lot slower. The beauty is getting information out that you can't get out of a paper chart. Data from Foley's catheters. Other data that one needs is only findable on a large scale through EHRs. On a minute to minute, it slows people down. The big issue is to develop a system, anything you take from the EHR is only as good as what's put in, and the person putting it in can make up the data. Developed a system in nursing flowsheet where there is a forced field for contact isolation precautions and generate a list and see if appropriately isolated and who can come off of. Every note, there is an infection control part of the flowsheet for nurses on do they have Foley's catheter and they don't put that in even if taking care of the patient. Again, depending on the hospital, more or less. Can say the same for the respirator days? Wonderful for a system to collect data and understand infection rates."

\section{Support for meeting U.S. Food and Drug Administration (FDA) paperwork requirements for investigational drugs.}

One SME suggested that EHRs could support meeting paperwork requirements from the FDA when using investigational drugs, which might occur regularly during an epidemic and could potentially slow down the provision of care in urgent situations. The requirements are already known, and therefore effort could be proactively expended now to optimize the workflow prior to needing the functionality on a regular basis.

Related statements from SMEs are:

- One expert from Hospital 3 stated: "There is an issue, however, with using investigational drugs in that there is required paperwork from the FDA in order to use them. It is not easy to record the paperwork in the EHR, and it's not always in the pharmacy in the same way as other medications. If we were regularly using investigational drugs such as during an epidemic, we would want functionality to enable meeting the FDA paperwork requirements. In the old EHR system we had, 
we had built-in pharmacy alerts with pop-ups. It won't order for you, but it has possible substitutes when an alternative medication is needed. This would be helpful."

\section{Decision support for clinical workers regarding when to consult ID specialists.}

Two SMEs identified that a useful area to explore would be to help frontline providers be aware of when to consult specialists in infectious disease, such as to be aware of new information from the field that might affect how screening questions should be asked and interpreted.

Related statements from SMEs are:

- One expert from Hospital 1 stated: "For the frontline workers, they have to know to consult ID to see the patient, and so we target the front line with town halls, educational options, online education, but still it's not always easy to gain their full attention until something like where the Ebola patient was treated in state. If there was some way to alert them to think about something differently based upon new information from the field, that would be helpful, again either upon login or tied to patients at higher risk for some reason, such as having a fever."

One expert from Hospital 2 stated: "Lots of forms. On our intranet where people can find them, distributed. People in the ED know where they are and ask the appropriate questions. Also on the Internet. Have it on the infection control site on the institution-specific Intranet. Might do better to call a hospital in the middle of Midwest and ask how they're handling it. Needs obviously different in different locations."

\section{Support for tracking locations of hot spots for outbreaks.}

One area where EHRs could potentially provide support would be in helping to determine geographic "hot spots" and visualize them based on available data or additional data fields.

Related statements from SMEs are:

- One expert from Hospital 1 stated: "If you wanted to use zip codes to identify where patients live, one issue is that rural zip codes cover a large geographic space, whereas city zip codes cover a small geographic space. Would it not be better to use addresses and calculate distances from them rather than zip codes? In theory, 'freeware' or other software applications (probably not Google maps, but there should be something that does this well) could be used to calculate distances for treating hospitals based on their addresses and patients' home and work addresses are already known. Perhaps search on GEOCODING to see what is used. If EHRs had a field for country or origin or language for patients, this might be able to be used in screeners or trackers."

- Investigator: "We have been talking primarily where there are one-fers e.g., the primed healthcare system is able to handle the isolated cases. However, what happens in the setting of a true epidemic where the health system's resources are stressed? We've been discussing when a person outside the geographic area of the health system has an elaborate process to control and contain transmission of infection. Measles is a different type of scenario that was brought up by you where there was a possibility of a local epidemic. How IT would help where it's a many-person type of case, where now the clinical system is stressed, where the resources to properly handle anything are pushed to an extreme. In that scenario, what different types of capabilities in an IT system would be needed? As a clinician, that also leads to burdensome things that make the data murkier. If designing a system in that situation, would there be certain capabilities that would be helpful to know that a person is a certain risk category for the disease? Who else intersected this person during their hospital stay?" Expert from Hospital 2 stated: "We spent hours doing that. If the system would say--, here is the list of contacts and queue them up for you would that be helpful to you... The answer to that is absolutely, but lots of issues. Different systems can do that better. I went to each OB clinic and got a list of who was there in a certain time frame and created lists from that. Communications issues, having an approved flash drive would be wonderful." 


\section{Support for communicating to the public about seriousness of preventive measures during outbreaks .}

One of the SMEs suggested that the EHR could facilitate public education -for instance, to better understand the difference between a gastrointestinal illness and influenza, and thus understand better the criticality of taking preventive measures to avoid the flu and avoid spreading to others.

Related statements from SMEs are:

- One expert from Hospital 1 stated: "With the flu, many patients mistakenly think that a GI illness is the flu, and thus don't get as concerned as they should be with flu outbreaks. A true flu is sick in bed for 3 days with a high fever and muscle aches where you are miserable and can't do anything. People do die from it, even now before it has mutated." 


\section{Conclusions}

The insights of the subject matter experts identified a wide range of opportunities to increase public safety by augmenting existing EHR functionality. Some of these opportunities include:

- Identifying a patient as being suspected or confirmed of having a highly infectious disease;

- Directly accessing 'read only' information from a prior organization about past medical history, labs, treatments;

- Obtaining an accurate immunization history without relying on patients to provide information;

- Having an "at a glance" summary of the status for an infectious patient; and

- Correctly placing patients transferred from the outside in coordination with other organizations.

These insights provide a first step in creating an effective infrastructure to better identify, diagnose, treat, and report about the status of infectious disease outbreaks in the United States. The lessons from this effort might also have implications beyond the treatment of highly infectious diseases. A primary insight is that users need EHR information to be reliable and applicable, including being able to view a summary of a concise narrative of relevant history, and an easily interpretable view of when vaccinations were provided against the expected immunization schedule. In order to achieve this goal, there needs to be confidence that the correct patient's record is open, that the information in the record is accurate and pertains to that patient, and that information that is used in graphical representations in structured fields is complete, accurate, and integrates information from multiple sources, including data from other EHR systems.

The EHR needs to be a reliable tool verified through standardized testing that optimizes clinical outcomes and public health awareness by giving the healthcare team comprehensive and trusted information that can be efficiently interpreted across role-based physical and cognitive workflow. 


\section{Acronyms}

BP - Blood Pressure

C. diff - Clostridium difficile

CDC - Centers for Disease Control and Prevention

ED - Emergency Department

EHR - Electronic Health Record

EMS - Emergency Medical Services

ER - Emergency Room

EVD - Ebola Virus Disease

FDA - U.S. Food and Drug Administration

GI - Gastrointestinal

HIPAA - Health Insurance Portability and Accountability Act

H1N1 - Hemagglutinin Type 1 Neurominidase Type 1

ICU - Intensive Care Unit

ID - Infectious Disease

MDR - Multidrug Resistance

MERS - Middle East Respiratory Syndrome

MICU - Medical Intensive Care Unit

MMR - Measles, Mumps, and Rubella

MRN - Medical Record Number

MRSA - Methicillin-resistant Staphylococcus aureus

PCR - Polymerase Chain Reaction

PHIN - Public Health Information Network

PPE - Personal Protective Equipment

RN - Registered Nurse

SARS - Severe Acute Respiratory Syndrome

SME - Subject Matter Expert

TB - Tuberculosis

VADS - Vocabulary Access Distribution System 


\section{References}

${ }^{1}$ C. Spencer, "Having and Fighting Ebola - Public Health Lessons from a Clinician Turned Patient," in N Engl J Med, March 19, 2015, 372:1089-1091.

${ }^{2} 2014$ Ebola Events: Texas Health Resources Shares Lessons Learned, Action Plans and Improvements. https://www.texashealth.org/pages/about\%20texas\%20health\%20resources/sharing-lessons-improvingperformance.aspx. Accessed September 112015.

${ }^{3}$ Khabbaz RF1, Moseley RR2, Steiner RJ3, Levitt AM2, Bell BP4. Challenges of infectious diseases in the USA. Lancet. 2014 Jul 5;384(9937):53-63. doi: 10.1016/S0140-6736(14)60890-4. Epub 2014 Jul 1 ${ }^{4}$ PHVS_CountriesWidespreadTransmission_EVD. https://phinvads.cdc.gov/vads/ViewValueSet.action?id=82FCE50A-4559-E411-9618-0017A477041A. Accessed September 11, 2015.

${ }^{5}$ The Perpetual Challenge of Infectious Diseases. Fauci AS1, Morens DM The perpetual challenge of infectious diseases. N Engl J Med. 2012 Feb 2;366(5):454-61. doi: 10.1056/NEJMra1 108296.

${ }^{6}$ R.E. Gliklich, N.A. Dreyer, M.B. Leavy, Registries for Evaluating Patient Outcomes: A User's Guide [Internet]. 3rd edition. Rockville (MD): Agency for Healthcare Research and Quality (US); 2014 Apr. 4. ${ }^{7}$ Data Elements for Registries. H. Poor, An Introduction to Signal Detection and Estimation. New York: Springer-Verlag, 1985, Ch. 4. 